\title{
Microarray analysis of gene expression by microdissected epidermis and dermis in mycosis fungoides and adult T-cell leukemia/lymphoma
}

\author{
KEIKO HASHIKAWA ${ }^{1-3}$, SHINICHIRO YASUMOTO ${ }^{1}$, KAZUTAKA NAKASHIMA ${ }^{2}$, FUMIKO ARAKAWA ${ }^{2}$, \\ JUNICHI KIYASU ${ }^{2}$, YOSHIZO KIMURA ${ }^{2}$, HIROSHI SARUTA ${ }^{1}$, TAKEKUNI NAKAMA ${ }^{1,4}$, KAORI YASUDA ${ }^{5}$, \\ KOSUKE TASHIRO $^{6}$, SATORU KUHARA ${ }^{6}$, TAKASHI HASHIMOTO ${ }^{1}$ and KOICHI OHSHIMA ${ }^{2}$ \\ Departments of ${ }^{1}$ Dermatology and ${ }^{2}$ Pathology, Kurume University School of Medicine; ${ }^{3}$ Department of Dermatology, \\ Asakura Medical Association Hospital; ${ }^{4}$ Department of Dermatology, Kurume University Medical Center; \\ ${ }^{5}$ Cell Innovator, Inc., Venture Business Laboratory of Kyushu University; ${ }^{6}$ Laboratory of Molecular \\ Gene Technics, Department of Genetic Resources Technology, Graduate School of Genetic \\ Resources Technology, Faculty of Agriculture, Kyushu University, Japan
}

Received March 17, 2014; Accepted May 14, 2014

DOI: $10.3892 /$ ijo.2014.2524

\begin{abstract}
The characteristic histopathological feature of mycosis fungoides (MF) and adult T-cell leukemia/lymphoma (ATLL) is epidermotropism. To identify the mechanism for epidermotropism of lymphoma cells, total RNAs were obtained from skin biopsies of epidermis and dermis of MF and ATLL patients by means of laser capture microdissection, and used for subsequent complementary DNA (cDNA) microarray experiments. This procedure has made it possible for us to observe and evaluate the regional environment of MF and ATLL. Hierarchical cluster analysis revealed that the cDNAs could be clearly differentiated into MF and ATLL. CCL27 was expressed in the dermis generated from keratinocytes, CCR4/CCR6/CCR7/CCR10/cutaneous lymphocyte-associated antigen (CLA) lymphoma cells in the dermis, and CCL21 in the extracellular matrix (stroma). Lymphotoxin (LT) $\beta$ and CCL21 expression was significantly higher and that of CCR10 relatively for MF, while CCR4 and CLA expression was relatively higher for ATLL. In the epithelium, keratinocytes expressed CCL20/CCL27, and lymphoma cells CCR4/CCR6/CCR10, while CCR4, CCR6, CCL20 and CCL27 expression was relatively higher for ATLL than MF. The dermis of MF, but not that of ATLL, showed correlation between CCR7 and CCL21. These findings support the suggestion that chemokines and chemokine receptors are involved in the pathogenesis of
\end{abstract}

Correspondence to: Dr Keiko Hashikawa, Department of Dermatology, Kurume University School of Medicine, 67 Asahimachi, Kurume, Fukuoka 830-0011, Japan

E-mail: hassy-maru@ymdmail.jp

Key words: microarray analysis, laser capture microdissection, epidermotropism, regulatory $\mathrm{T}$ cell
MF and ATLL, indicate that cutaneous homing seems to be different for MF and ATLL, and point to the possibility that cutaneous T-cell lymphomas originate in regulatory $\mathrm{T}$ cells, especially in the case of ATLL.

\section{Introduction}

Mycosis fungoides (MF) is the most common form of cutaneous T-cell lymphomas (CTCL), which is a heterogeneous group of non-Hodgkin's lymphomas thought to result from malignancies of skin homing T cells (1) and presenting with patches, plaques and tumors.

While the early stage shows a wide clinical spectrum that overlaps with several inflammatory dermatoses, clinical as well as pathological findings indicate various overlaps between MF and inflammatory diseases, with epidermotropism disproportionate to the degree of spongiosis being one of the most useful pathological distinguishing features.

Adult T-cell leukemia/lymphoma (ATLL) is a human malignancy associated with human T-cell lymphotropic virus-type I (HTLV-1). Cutaneous lesions of ATLL consist of papules and nodules/tumors. While survival of ATLL cases with skin manifestations is reportedly significantly shorter than that of MF cases (2), histological findings for the two diseases are similar.

The mechanism of epidermotrophism seen in MF and ATLL has not yet been clearly identified. What is known is that chemokines regulate multiple cell functions, including cell chemotaxis, proliferation and apoptosis, and are involved in leukocyte transendothelial migration and homing to tissues. Previous studies have reported that positivity for CCR4 is significantly associated with skin involvement in MF and ATLL, and CCR10 is expressed by malignant cells in MF and Sézary syndrome (SS) (3). Malignant MF and SS cells also show high expression of CCR7. The cutaneous lymphocyteassociated antigen (CLA) recognized by the HECA-452 
antibody is an adhesion molecule selectively expressed by a subset of circulating memory T-cells, normal $\mathrm{T}$ cells in inflamed skin and by the vast majority of CTCL (4-6). A previous study found that CLA was expressed on epidermotrophic lymphoma cells in all early stages MF. As for ATLL, $\mathrm{CLA}^{+}$cases showed a significant preference for skin involvement when compared with the CLA ${ }^{-}$cases as also previously reported by other investigators (7).

Complementary DNA (cDNA) microarray technology is a powerful tool for gaining insight into the molecular complexity and pathogenesis of various diseases and makes it possible to identify differences in numerous gene expressions.

Since the skin consists of epidermis [including keratinocytes and dendritic cells (DCs)], dermis (stroma and vessels) and subcutaneous tissue with or without inflamed and/or inflammatory cells, gene expression of epidermis and dermis should be analyzed separately to gain a better understanding of the contribution made by each component of a tissue to the pathogenesis of epidermotrophism. To this end, laser capture microdissection (LMD) was used in our study to allow us to focus on the differences in gene expression levels between epidermis and dermis in MF and ATLL and to subsequently determine these expressions immunohistochemically in patients.

It is believed that this technique, which combines LMD and microarray technology for the analysis of CTCL gene expression in epidermis and dermis, can improve our understanding of epidermotrophism at the molecular level.

\section{Materials and methods}

Eighteen skin samples obtained from patients treated at the Department of Dermatology, Kurume University School of Medicine consisted of five epidermal and three dermal samples each from MF and ATLL as well as two epidermal samples from dermatitis patients used as controls. Skin biopsies were performed after informed consent had been obtained from all patients or their guardians in accordance with the Declaration of Helsinki. Both paraffin-embedded and frozen tissues were used. Histopathological diagnoses were based on the World Health Organization classification (WHO) and performed by five pathologists (K.H., J.K., Y.K., H.S. and K.O.). The clinical data for all patients obtained from the medical records are summarized in Table I, which shows that both epidermal and dermal samples were harvested from two MF (E-M3/D-M1 and E-M5/D-M2) and two ATLL (E-A2/D-A1 and E-A3/D-A2) patients. The other samples were either epidermal or dermal. This study was approved by the Kurume University Institutional Review Board.

Laser microdissection. The samples were embedded in an optical cutting temperature compound (Sakura Finetechnical, Tokyo,Japan), immediately frozen in liquid nitrogen, and stored at $-80^{\circ} \mathrm{C}$ for microdissection. A series of $10-\mu \mathrm{m}$ thick sections were cut from frozen tissue specimens at $-20^{\circ} \mathrm{C}$, and mounted on 2.0- $\mu$ m-thick PEN-Membrane slides (MicroDissect GmbH, Herborn, Germany). The epidermal and/or dermal regions were microdissected from the about 70 cryosections by means of LMD. After fixation in $100 \%$ ethanol, the slides were stained in turn with Mayer hematoxylin and eosin, washed in diethylpyrocarbonate-treated water at each phase of the process and then air-dried with a fan. The frozen sections were microdissected with a Leica LMD6000 laser microdissection system (Leica, Wetzlar, Germany) in accordance with the company's protocol. Finally, the microdissected fragments were dropped into $0.5 \mathrm{ml}$ tube caps containing $50 \mu \mathrm{l}$ lysis buffer for RNA extraction (8).

RNA extraction and biotinylated cRNA amplification. Total RNA was extracted from the samples collected by means of LMD and with an RNAqueous-Micro kit (AM1931; Ambion, Austin, TX) according to the manufacturer's instructions. Complementary RNA (cRNA) amplification and labeling with biotin were used for gene expression profiling by microarray analysis. Briefly, $500 \mathrm{ng}$ total RNA was amplified overnight (14 h) with the Illumina Total Prep RNA Amplification kit (AMIL1791; Ambion) according to the manufacturer's protocol. Reaction cRNA was biotinylated during in vitro transcription.

Illumina BeadChips microarray. Sentrix Human WG-6 v3.0 Expression BeadChips were purchased from Illumina, Inc. (San Diego, CA). More than 48,000 different bead types, each with a 50-base gene-specific probe, are represented on a single Beadchip. For each probe represented on the array, beads are assembled with an average 30-fold redundancy. A hybridization mixture containing $1.5 \mu \mathrm{g}$ biotinylated cRNA was hybridized to the beadchips at $58^{\circ} \mathrm{C}$ overnight $(18 \mathrm{~h})$ before being washed and stained with streptavidin-Cyanine-3 (Cy3) (PA23031; GE Healthcare, Buckinghamshire, UK) according to the manufacturer's protocol. Beadchips were scanned on Illumina BeadStation 500 and fluorescent hybridization signals were assessed with Illumina Beadstudio software.

The data discussed in this publication have been deposited in National Center for Biotechnology Information (NCBIs) Gene Expression Omnibus (GEO, http://www.ncbi.nlm.nih. gov/geo/) and are accessible through GEO Series accession number GSE40639.

Statistical analysis. For the pre-processing step, variance in the data was first stabilized with the variance stabilizing transform method (9) and then normalized with a robust spline normalization method, both of which are used with the Lumi BioConductor package (Illumina) (10). Effectively absent transcripts were filtered out to reduce false positives. Detection of transcripts was considered to be achieved if the detection p-value calculated from the background with the Illumina BeadStudio was $<0.05$ for all hybridizations. The Significance Analysis of the Microarrays statistical test (11), which is used as part of the bioconductor 'samr' package and takes multiple testing into account by estimating the false discovery rate, was used to identify differentially expressed transcripts for a comparison of MF and ATLL patients. If a transcript was up- or downregulated by a factor of $\geq 2$ and had a Significance Analysis of Microarrays q-value (false discovery rate) of $<0.001$, we regarded the expression of this transcript in MF patients as different from that in ATLL patients. To obtain reproducible clusters for classifying the 16 samples (all samples detailed above except for the two control samples), expression data were analyzed with 
Table I. Summary of patients enrolled in this study: number, age, gender and stage.

\begin{tabular}{lclcc}
\hline Sample no. & Age (years) & Gender & TNM & Stage \\
\hline MF & & & & \\
E-M1 & 78 & Male & T2aNOM0 & IB \\
E-M2 & 62 & Female & T2bN0M0 & IB \\
E-M3,D-M1 & 57 & Male & T1bN0M0 & IA \\
E-M4 & 22 & Male & T1bN0M0 & IA \\
E-M5, D-M2 & 64 & Male & T2bNOM0 & IB \\
D-M3 & 61 & Male & T2bN0M0 & IB \\
ATLL & & & & \\
E-A1 & 56 & Male & T2aN2M0 & IIA \\
E-A2, D-A1 & 70 & Male & T2cN0M0 & IB \\
E-A3, D-A2 & 32 & Female & T2cN0M0 & IB \\
E-A4 & 63 & Male & T3aNxM0 & IIB \\
E-A5 & 79 & Male & T3bN0M0 & IIB \\
D-A3 & 54 & Male & T3aN1M0 & IIB \\
Control & & & & \\
N1 & 66 & Male & & \\
N2 & 71 & Female & & \\
\hline
\end{tabular}

The samples were obtained from six MF and ATLL patients each, and from two dermatitis patients as controls. The five epidermal (E) samples of MF (-M) patients were numbered E-M1 through E-M5, and the three dermal (D) samples of MF (-M) patients were numbered D-M1 through D-M3. Similarly, the ATLL (-A) samples were numbered E-A1 through E-A5 and D-A1 through D-A3. The two epidermal samples from dermatitis patients used as controls were numbered N1 and N2.

GeneSpring 7.2 software (Silicon Genetics, Redwood City, CA), which was also used to generate heatmaps of certain genes of interest.

Immunohistochemistry. The paraffin-embedded specimens were used for manual immunohistochemical analysis of CCR4 (Pharmingen, San Diego, CA), CCR7, CCR6 and CCR10 (all from Medical and Biological Laboratories Co., Ltd., Nagoya, Japan), CCL20, CCL27, lymphotoxin (LT) $\beta$ and TNF receptor (TNFR) 2 (all from R\&D Systems, Minneapolis, MN), CCL21 (Santa Cruz Biotechnology, Santa Cruz, CA), $\beta$-defensin (BDF) 1 (Phoenix Pharmaceuticals, Inc., Burlingame, CA) as previously described (12). Appropriate positive and negative control experiments were run simultaneously. Heat-mediated antigen retrieval was used for all analyses except those of CCR6 and BDF1. The staining results were evaluated semiquantitatively by three independent observers, and scored comprehensively in view of intensity, expression pattern and number of positive cells, and so on. Images were visualized with an Olympus AX80 microscope (Olympus, Tokyo, Japan), equipped with an Olympus Planapo 40x/0.95 numerical aperture objective. Images were captured with an Olympus DP70 camera and
Olympus DP controller software, and were processed with Olympus DP manager software.

\section{Results}

Following appropriate normalization and standardization procedures, the data on each chip were compared with each other by using a hierarchical clustering method (Fig. 1). Genes differentially expressed in MF and ATLL were organized by means of Ingenuity Pathway Analysis into an interactome network, which was then used for an comparison of the gene expression (transcriptional profile) of epidermal or dermal MF with that of ATLL. In the epidermis, 580 probe sets were identified as upregulated or downregulated on a heat map (Fig. 1A), while in the dermis, 234 probe sets were found to be up- or downregulated (Fig. 1B). The first 10 genes showing the highest upregulation are listed in Table II. In the dermis, LT $\beta$ and CCL21 in particular showed different expressions in MF and ATLL.

Next, we focused on the array data that could identify the possible involvement of specific chemokine receptors and ligands, including CCR4, CCR6, CCR7 and CCR10 and others, in the pathophysiology of CTCL, MF and ATLL. CCR4 expression was especially high in ATLL, while CCL27 expression in the epidermis was high in both MF and ATLL, and CCR10, a receptor of CCL27 detected in the dermis, was more highly expressed in MF than in ATLL. Also in the dermis, expression of the LN homing molecule CCR7 was high in both MF and ATLL, while a close correlation between CCR7 and CCL21, a chemokine receptor and its ligand, was observed in MF. CCR6 expression was elevated in the epidermis and dermis of both MF and ATLL, while expression of CCL20, a CCR6 ligand, showed no correlation with CCR6. BDFs were identified as not only antibacterial peptides but also as CCR6 ligands. BDF 1 expression was reduced in the epidermis of MF and ATLL, while BDF 3 expression in the epidermis of MF was higher than in controls, which was similar to the finding of a previous study (13).

We also focused on CLA, which is recognized as an adhesion molecule selectivity expressed by a subset of circulating memory $\mathrm{T}$-cells, normal $\mathrm{T}$ cells in inflamed skin and by the vast majority of CTCL (4-6). CLA was expressed in the dermis of both MF and ATLL, with a particularly high expression in ATLL.

In order to confirm the expression levels of the proteins, CCR6, CCR7, CCR10, CCL20, CCL21, CCL27, BDF1, LT $\beta$ and TNFR2 on MF and ATLL tissue sections were subjected to immunohistochemistry. The findings of the immunohistochemical analysis were basically consistent with the results of the microarray analysis. A representative stain is shown in Fig. 2 and the immunohistochemical scores are presented graphically in Fig. 3.

In principle, CCR4, CCR6, CCR7, CCR10 and/or CLA were all expressed in the lymphoma cells. CCR4, CCR6 and CCR10 were identified in both dermis and epidermis, while CCL27 and CCL20 were expressed in keratinocytes and CCL21 was expressed in the extracellular matrix (stroma). In addition, CCL27 was also stained in dermis released from keratinocytes.

In controls, epidermal keratinocytes weakly expressed CCL27 within their cytoplasm, and mainly in the spinous 


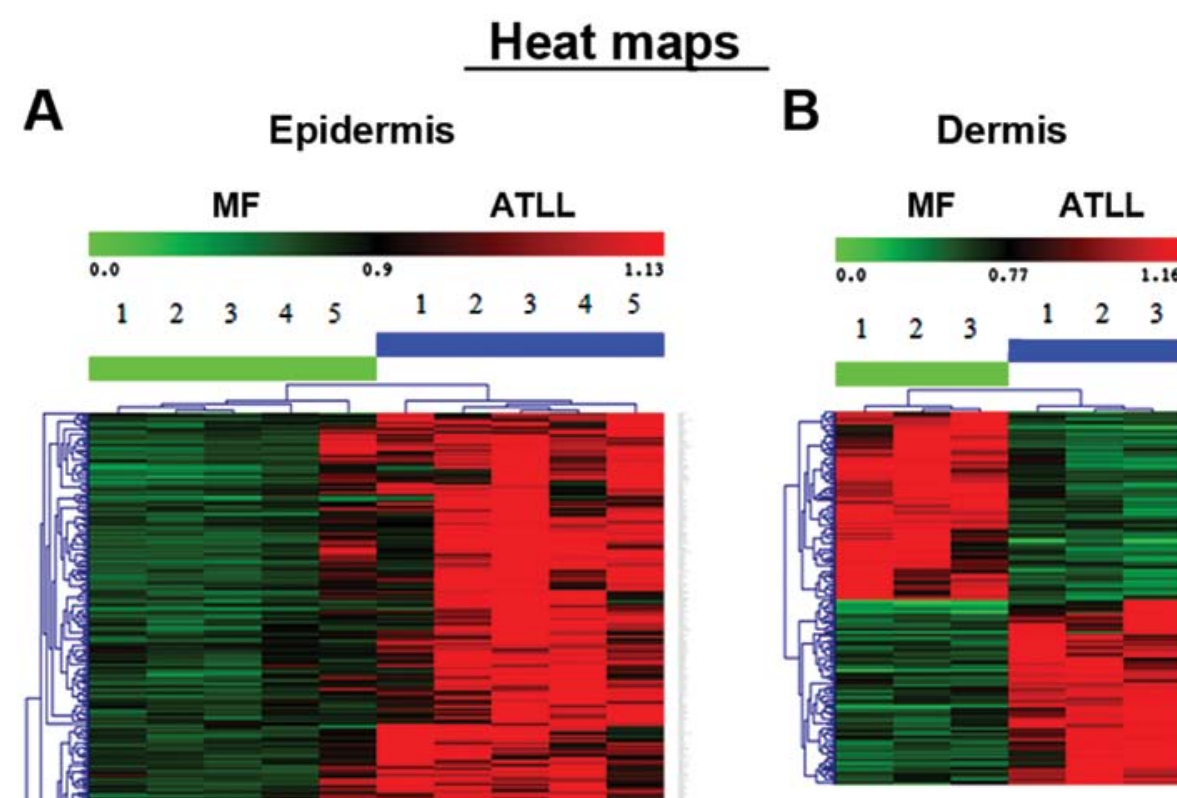

234 probes

Figure 1. Gene expression profiles and clustering. The gene expression of MF was compared with that of ATLL by using a hierarchical clustering method. (A) In the epidermis, 580 probe sets were identified as up- or downregulated. (B) In the dermis, 234 probe sets were identified.

layers of the epidermis. However, positive lesions of epidermis keratinocytes were stronger in thicker layers in most MF and ATLL patients than in controls. Moreover, CCL27 was expressed not only in cytoplasm but also occasionally in membrane of MF and ATLL, so that there was in fact not much difference between these two types of CTCL. Faint CCL27 expression in dermis was detected in MF, ATLL and in one of the controls. The differences in CCL27 expression levels in dermis were immunohistochemically imperceptible. CCR10 was stained weakly in both epidermis and dermis, but in the dermis usually more strongly in MF than in ATLL cells, which correlated moderately with the overall gene expression profile. CCR6 expression was more elevated in the epidermis and dermis of both MF and ATLL than in controls, and the immunohistochemical score correlated with the result of the microarray analysis. CCL20 was expressed very weakly in the cytoplasm of keratinocytes, stained in the granular layer of all samples and in all layers of the epidermis of some samples.

Although each one of the controls and MF samples showed high levels of CCL20, all ATLL samples displayed high expression in the epidermis. Expression of BDF1 in nucleus and/or nuclear membrane was diminished in the epidermis of MF and ATLL. Because the stain was quite faint, it was difficult to determine even whether CCL20 and BDF1 in the dermis were positive or negative. The cell membrane of infiltrating lymphoma cells stained for CCR7, while staining for CCL21 was observed within the cytoplasm of epidermal keratinocytes and was diffusely distributed on the dermal extracellular matrix. The intensity and density of $\mathrm{CCR} 7^{+}$ infiltrating lymphoma cells correlated with the intensity of CCL21 in stroma in the dermis of MF cases. LT $\beta$ positive cells were scattered throughout the dermis and their expression was homogeneous in the nucleus or the surface of the nucleus of lymphoma cells, while LT $\beta$ expression levels in the dermis were higher for MF than for ATLL. No correlation with LT $\beta$ was observed for TNFR2, which is one of the soluble secreted LT homotrimers (LT $\alpha 3)$ that trigger TNFRs.

\section{Discussion}

The microarray and immunohistochemistry findings of our study showed high expression of LT $\beta$ and CCL21, and correl- 
Table II. Genes differentially regulated in MF and ATLL.

A, Upregulated genes in MF compared with genes in ATLL

\begin{tabular}{|c|c|c|c|}
\hline Epidermis & Gene symbol & Gene name & Exp. value \\
\hline 1 & ZSCAN18 & Zinc finger and SCAN domain containing 18 & 1.392 \\
\hline 2 & COL16A1 & Collagen & 1.217 \\
\hline 3 & EID2B & EP300 interacting inhibitor of differentiation 2B & 1.167 \\
\hline 4 & HSPA1A & Heat shock $70 \mathrm{kDa}$ protein $1 \mathrm{~A}$ & 1.046 \\
\hline 5 & GGA1 & Golgi associated & 1.038 \\
\hline 6 & LRIG1 & Leucine-rich repeats and immunoglobulin-like domains 1 & 1.010 \\
\hline 7 & XPNPEP3 & X-prolyl aminopeptidase (aminopeptidase P) 3 & 1.003 \\
\hline 8 & SMARCA2 & SWI/SNF related & 0.979 \\
\hline 9 & ID2 & Inhibitor of DNA binding 2 & 0.968 \\
\hline 10 & ARL6IP5 & ADP-ribosylation-like factor 6 interacting protein 5 & 0.950 \\
\hline Dermis & Gene symbol & Gene name & Exp. value \\
\hline 1 & LTB & Lymphotoxin $\beta$ & 2.946 \\
\hline 2 & CCL21 & Chemokine (C-C motif) ligand 21 & 2.189 \\
\hline 3 & IFITM3 & Interferon induced transmembrane protein 3 & 1.666 \\
\hline 4 & THY1 & Thy- 1 cell surface antigen & 1.594 \\
\hline 5 & ACTN1 & Actinin & 1.585 \\
\hline 6 & CHST15 & Carbohydrate & 1.449 \\
\hline 7 & PON2 & Paraoxonase 2 & 1.447 \\
\hline 8 & CYR61 & Cysteine-rich & 1.436 \\
\hline 9 & TAGLN & Transgelin & 1.436 \\
\hline 10 & PRRX1 & Paired related homeobox 1 & 1.412 \\
\hline
\end{tabular}

B, Downregulated genes in MF compared with genes in ATLL

\begin{tabular}{|c|c|c|c|}
\hline Epidermis & Gene symbol & Gene name & Exp. value \\
\hline 1 & GPX3 & Glutathione peroxidase 3 & -2.097 \\
\hline 2 & PSMC1 & Proteasome & -1.668 \\
\hline 3 & CALML3 & Calmodulin-like 3 & -1.587 \\
\hline 4 & NDRG4 & NDRG family member 4 & -1.169 \\
\hline 5 & PRSS3 & Protease & -1.113 \\
\hline 6 & ADM & Adrenomedullin & -1.063 \\
\hline 7 & SMOX & Spermine oxidase & -1.056 \\
\hline 8 & CBX3 & Chromobox homolog 3 & -1.044 \\
\hline 9 & WBP5 & WW domain binding protein 5 & -0.970 \\
\hline 10 & LRRC20 & Leucine rich repeat containing 20 & -0.967 \\
\hline Dermis & Gene symbol & Gene name & Exp. value \\
\hline 1 & TOX2 & TOX high mobility group box family member 2 & -2.925 \\
\hline 2 & ADAP1 & ArfGAP with dual PH domains 1 & -2.848 \\
\hline 3 & AKAP7 & A kinase anchor protein 7 & -2.449 \\
\hline 4 & CADM1 & Cell adhesion molecule 1 & -2.293 \\
\hline 5 & ZNF365 & Zinc finger protein 365 & -2.157 \\
\hline 6 & RGS2 & Regulator of G-protein signaling 2 & -2.129 \\
\hline 7 & MTX3 & Metaxin 3 & -2.080 \\
\hline 8 & SH3KBP1 & SH3-domain kinase binding protein 1 & -1.871 \\
\hline 9 & CHST7 & Carbohydrate sulfotransferase 7 & -1.792 \\
\hline 10 & CST7 & Cystatin F (leukocystatin) & -1.739 \\
\hline
\end{tabular}

The 10 most significant signatures of gene expression are shown. LT $\beta$ and CCL21 in the dermis showed higher expressions in MF than in ATLL. 


\section{Immunohistochemistry}

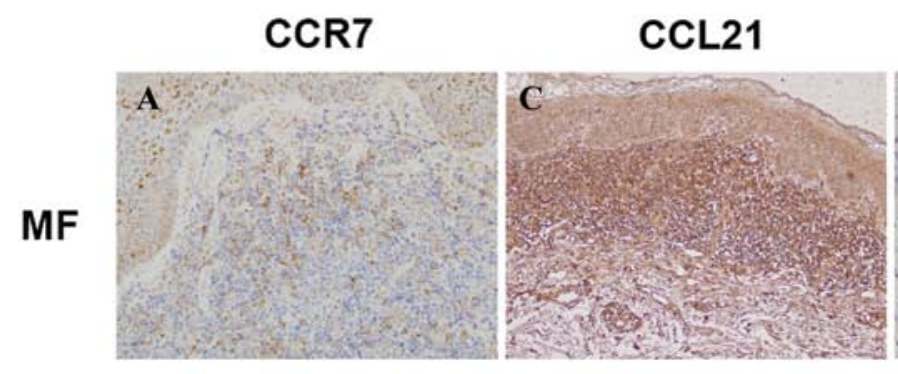

(E-M5 D-M2)

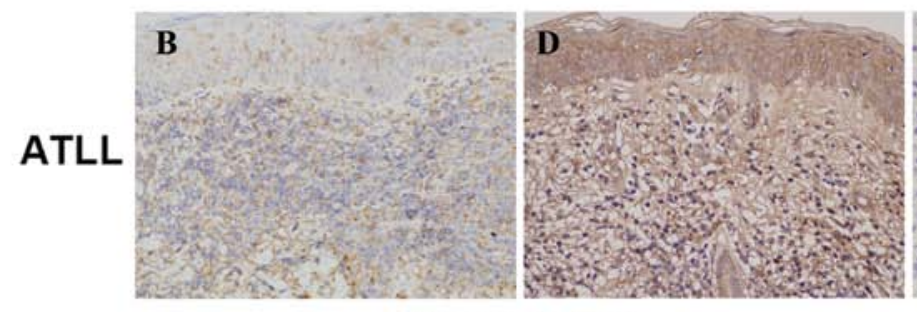

(E-A3 D-A2)

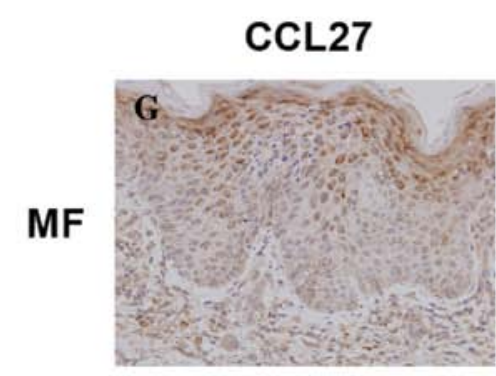

(E-M1)

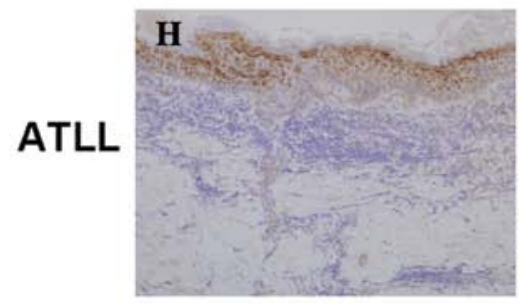

(E-A3 D-A2)
CCR6

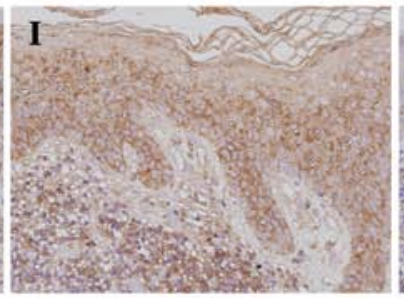

(E-M5 D-2)
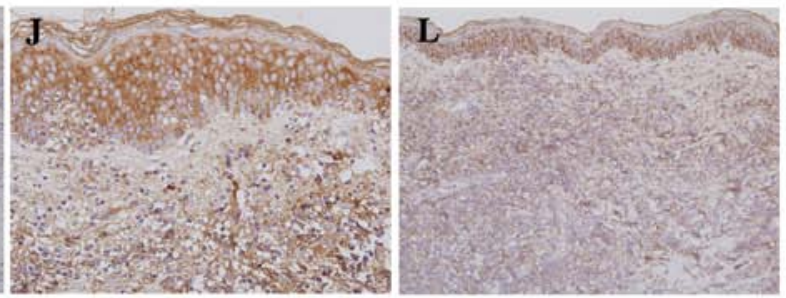

(E-A2 D-A1)

Figure 2. Representative immunohistochemical stain. CCR7 was expressed in the lymphoma cells (A, B) and CCL21 in the extracellular matrix (stroma) $(C, D)$. CCL21 and LT $\beta$ were higher in the dermis in MF (C, E) than in ATLL (D, F). CCL27 (G-H) and CCL20 (K-L) were expressed in keratinocytes. CCR6 expression in the epidermis and dermis was elevated in both MF and ATLL (I-J). CCL27, CCR6 and CCL20 in the epidermis were relatively higher in ATLL than in MF. (Original magnification x100). (A, C, I, K) were from sample no. E-M5 D-M2 and (B, D, H) from E-A3 D-A2, (E) was from D-M3, (F, J, L) were from E-A2 D-A1 and $(\mathrm{G})$ was from E-M1.

tion between CCL21 and CCR7, a receptor of CCL21 which is the LN homing molecule, was observed in the dermis of MF. CCR7 expression was also high in the dermis of ATLL.

LT $\beta$, which was indentified in the dermis of MF samples in our study, is a member of the tumor necrosis factor superfamily. LT $\beta$ in human cells contains a transmembrane domain, and the surface LT form is most likely a trimer with an LT $\alpha 1 \beta 2$ stoichiometry, while the LT $\alpha 1 \beta 2$ heterotrimer binds the LT $\beta$ receptor (LT $\beta R$ ) (14).

LTs are expressed by activated T-, B-, NK- and lymphoid tissue inducer cells (15-17) and are crucial for organogenesis and maintenance of lymphoid tissues $(18,19)$. LT $\beta R$, on the other hand, is expressed on many different cell types including cells of epithelial lineages, while ligation of LT $\beta R$ results in $\mathrm{NF}-\kappa \mathrm{B}$ activation (20-22) and leads to secondary lymphoid organogenesis and homeostasis. Signaling via LT $\beta$ R is involved not only in host defense and autoimmune diseases, but also in tumor cell proliferation (23). Furthermore, NF- $\kappa$ B stimulates proliferation and blocks programmed cell death (apoptosis) in various cell types $(24,25)$.

Previous reports have suggested that the activation of the $N F-\kappa B$ signaling pathway via LT $\beta R$ results in the prolifer- 

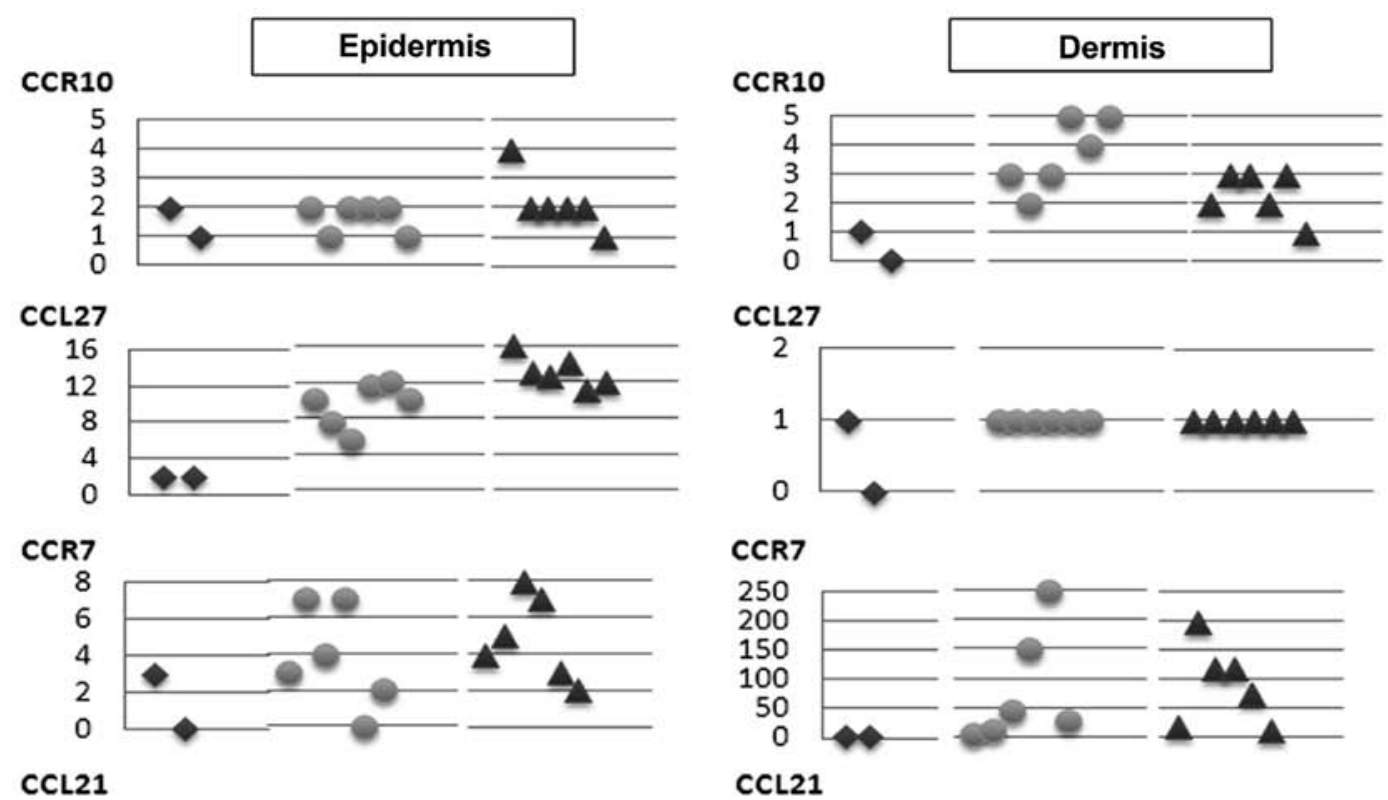

CCL21
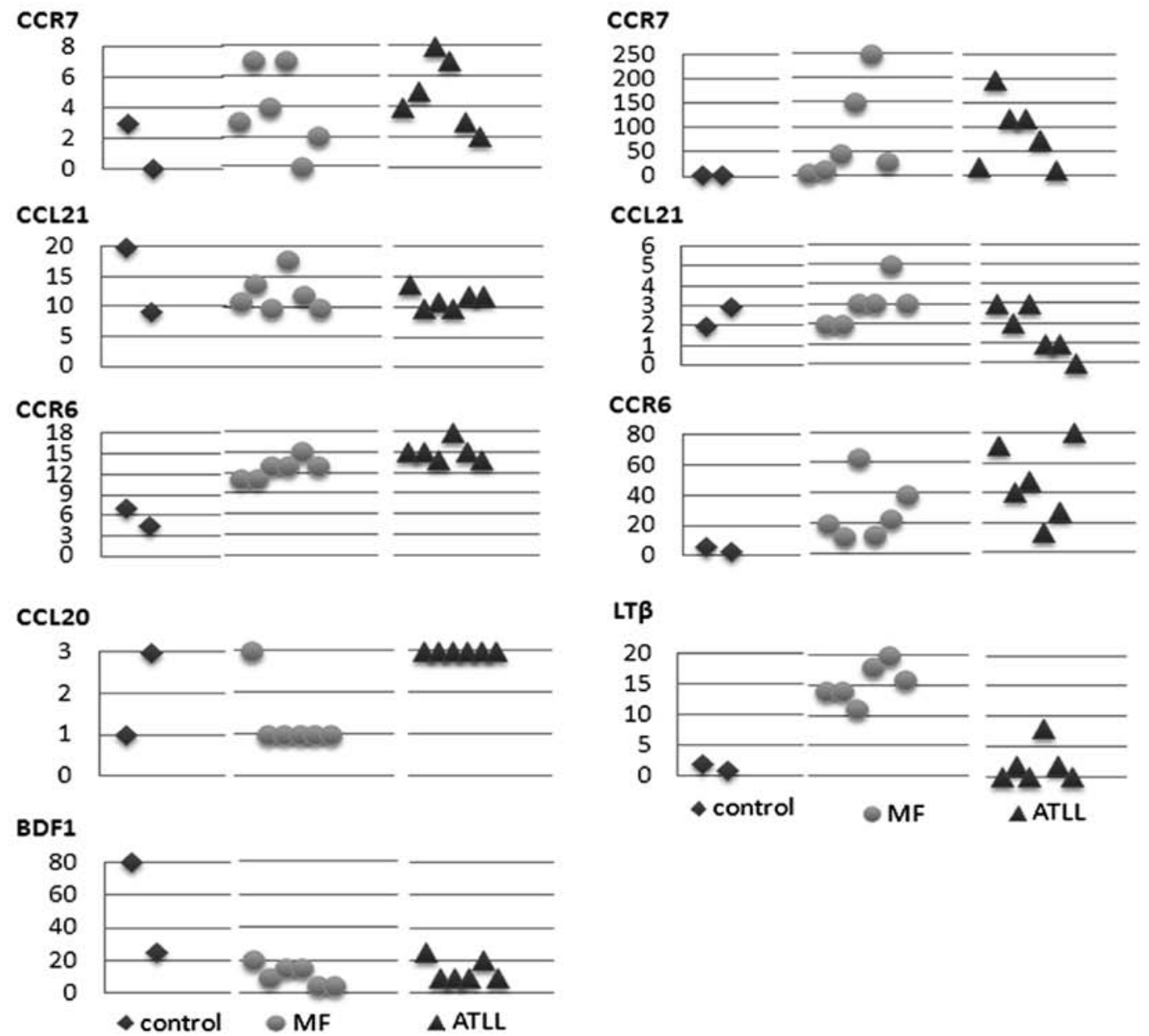

Figure 3. Immunohistochemical scores. Scores for LT $\beta$ and CCR10 in the dermis were usually higher for MF than ATLL. CCR6 was more elevated in the epidermis as well as dermis of both MF and ATLL than of controls. CCL20 showed a high score in the epidermis of all ATLL samples. Controls are shown as rhomboids, MF cases as dots and ATLLs as triangles.

ation of melanoma (26) and hepatocellular carcinoma (27). $\mathrm{NF}-\kappa \mathrm{B}$ activation was also detected in lymphoid malignancies $(28,29)$. In this connection, it was found that peripheral $\mathrm{T}$ cell lymphomas/not otherwise specified (PTCLs/NOS) with NF- $\mathrm{KB}$ inactivity showed better survival (30-32).

Another study reported that LT $\beta$ R organizes the recruitment and survival of CD4 T cells through the induction of CCL21 expression which is produced exclusively by stroma (33). In our study we found that expression levels of both the LT $\beta$ and CCL21 gene are high in the dermis of MF. These findings seem to suggest that LT $\beta R$, which can be expressed in epidermis, organizes the recruitment and survival of CD4-positive MF cells through the induction of CCL21.

CCR7 (the receptor of CCL21) physiologically regulates the lymphoid homing of T-cells and probably plays an important role in the tropism of CTCL cells to peripheral LNs, which constitutively synthesize CCR7 ligands, CCL19 and CCL21 (34). It has been further hypothesized that CCR7, which is expressed at fairly high levels in SS cells (35), may function as a mediator of LN infiltration of CTCL. In our study, CCR7 expression was detected in the dermis of MF and ATLL. Interaction between CCR7 and CCL21 in the dermis 
of MF probably functions to maintain localization to the skin for a long time. On the other hand, T cells infected by HTLV-1 circulate in the bloodstream from the outset, while ATLL cells, expressing the skin-homing properties of CCR4, CLA, CCR6 and CCR10, may affect cutaneous involvement in smorderling or cutaneous type of ATLL patients.

While T cells infected by HTLV-1 are circulating in the peripheral blood, they can spontaneously encounter CCL21 produced by $\mathrm{LNs}$, and $\mathrm{CCR} 7^{+}$ATLL cells can be trapped in the LNs. Migration to the LNs can thus occur at an earlier stage in ATLL than in MF.

CTCL is characterized by clonal expansion of a mature CD4-positive clone of the Th phenotype, putatively from a skin homing subset of memory T cells (36) whose migration to respective tissues is tightly regulated by adhesion molecules and chemokine receptors. For example, memory $\mathrm{T}$ cells that infiltrate the skin express a unique adhesion molecule known as CLA. Chemokines and their receptors have also been associated with tumor metastasis (37-39) and possibly trafficking of lymphoma cells (40). In addition, chemokines are produced by not only malignant $\mathrm{T}$ cells but also epidermal keratinocytes, DCs and dermal vessels. We therefore did not use fractionated malignant $\mathrm{T}$ cells, but the microdissected epidermal and dermal affected regions in MF and ATLL to investigate the expression profiles of CCR4, CCR6, CCR7, CCR10, CCL20, CCL21, CCL27, BDFs and CLA by means of microarray analysis and/or immunohistochemistry. We found that CCL27 expression in the epidermis was high in both MF and ATLL. CCR10 expression observed in the dermis was higher in MF than ATLL, and the microarray analysis showed that CCR4 expression was especially high in ATLL. Previous studies have reported that CCR4 expression was detected much more frequently in both MF and ATLL than in healthy controls $(41,42)$.

In addition, findings of a previous study indicated that DCs synthesize CCR4 ligands, which rapidly stimulate chemotaxis of, or conjugate formation with, normal T cells $(43,44)$. A characteristic histopathological marker, Pautrier's microabscesses of CTCL, which are known to be DC-malignant T cell conjugates (45), may be initiated by DC-derived chemokines. Other data indicated that preferential expression of CCR4 constitutes a sign of worse prognosis, and CCR4 expression was especially high in ATLL in our study. This finding corresponds to the pathological feature of more visible and larger Pautrier's microabscesses as well as the clinical feature of worse prognosis for ATLL than for MF.

CCR10 is enriched in $\mathrm{CLA}^{+}$skin-homing T cells of psoriasis, atopic dermatitis and CTCL patients, while it is only rarely expressed on peripheral blood $\mathrm{T}$ cells and skin samples from healthy persons $(3,46)$. Moreover, CCL27 (CCR10 ligand) is constitutively present in epidermal keratinocytes (basal layer) under basal, non-inflammatory conditions and can be rapidly released from activated keratinocytes (46). CCL27-CCR10 interactions thus play an early role in the pathophysiology of MF from the patch stage (47), and an increase in CCL27 in the serum of MF patients was observed in previous studies (48). Our data for CCL27-CCR10 interactions agreed with the pathophysiology outlined above.

Our findings also indicate that CCR4 in ATLL as well as CCR10 in MF may play a much more prominent role in epidermotrophism. However, the origin of malignant $\mathrm{T}$ cells in MF and ATLL remains to be fully elucidated. High-level expressions of CCR4, CLA and CCR6 indicate the possibility of not only Th but also Treg origin especially in ATLL. Treg action suppresses activation of the immune system and tolerance for self-antigens. The phenotype of $\mathrm{CD} 4{ }^{+} \mathrm{CD} 25^{+}$and the expression of Foxp3, a master transcriptional regulator of Treg development and function, suggest that human skin-resident $\mathrm{T}$ cells appear to represent Tregs. Tregs also express functional skin-homing receptors. Although CCR6 does not seem to be a functional marker for Treg, it may facilitate $\mathrm{CLA}^{+}$Treg migration to skin. As for Th, there are conflicting reports about Th1 and Th2 profiles of CCR4 in MF. Berger et al demonstrated that malignant $\mathrm{CD}^{+}{ }^{+} \mathrm{T}$ cells can be induced to express a CD25+ Treg phenotype (49). Our analyses, however, disclosed that CCR4 and CCR6 in both epidermis and dermis and CLA in dermis were expressed in MF and ATLL, with the expression being especially high in ATLL. Our data therefore support the possibility that the origin of CTCL is Treg (particularly in ATLL) as also previously reported (50). Further investigation will be required to determine whether CTCL shows Th1/Th2/Treg-type polarization in lesional skin.

While the mechanisms involved in tumor homing have not yet been fully identified, it has been suggested that chemokines and chemokine receptors are involved in the pathogenesis of tumor homing. There are no previous reports on the use of the microdissection method for a comparison of epidermal and dermal gene expression levels in MF and ATLL by means of microarray analysis, a procedure which has made it possible to observe and evaluate the regional environment of MF and ATLL, including malignant and inflamed T-cells, DCs, keratinocytes and dermal vessels. DNA microarray analysis enabled us to comprehensively identify differences and patterns in gene expression in MF and ATLL, while our findings support the notion that CCR10 and its ligand CCL27 may contribute to the skin infiltration of malignant T-cells in MF and ATLL. However, further studies are needed to clarify the mechanism for epidermotrophism because of the complexity of its interactions.

\section{Acknowledgements}

The authors would like to thank Konomi Takasu, Mayumi Miura and Kanoko Miyazaki for their technical support.

\section{References}

1. Willemze R, Jaffe ES, Burg G, et al: WHO-EORTC classification for cutaneous lymphomas. Blood 105: 3768-3785, 2009.

2. Kikuchi A, Ohata Y, Matsumoto H, Sugiura M and Nishikawa T: Anti-HTLV-1 antibody positive cutaneous T-cell lymphoma. Cancer 79: 269-274, 1997

3. Notohamiprodjo M, Segerer S, Huss R, et al: CCR10 is expressed in cutaneous T-cell lymphoma. Int J Cancer 115: 641-647, 2005.

4. Picker LJ, Terstappen LW, Rott LS, Streeter PR, Stein H and Butcher EC: Differential expression of homing-associated adhesion molecules by T-cell subsets in man. J Immunol 145: 3247-3255, 1990

5. Picker LJ, Michie SA, Rott LS and Butcher EC: A unique phenotype of skin-associated lymphocytes in humans: preferential expression of the HECA-452 epitope by benign and malignant T cells at cutaneous sites. Am J Pathol 136: 1053-1068, 1990. 
6. Berg EL, Yoshino T, Rott LS, et al: The cutaneous lymphocyte antigen is a skin lymphocyte homing receptor for the vascular lectin endothelial cell leukocyte adhesion molecule 1. J Exp Med 174: 1461-1466, 1991.

7. Tanaka Y, Wake A, Horgan KJ, et al: Distinct phenotype of leukemic $\mathrm{T}$ cells with various tissues tropisms. J Immunol 158: 3822-3829, 1997.

8. Rubin MA: Use of laser capture microdissection, cDNA microarrays, and tissue microarrays in advancing our understanding of prostate cancer. J Pathol 195: 80-86, 2001.

9. Lin SM, Du P, Huber W and Kibbe WA: Model-based variancestabilizing transformation for Illumina microarray data. Nucleic Acids Res 36: e11, 2008.

10. Du P, Kibbe WA and Lin SM: lumi: a pipeline for processing Illumina microarray. Bioinformatics 24: 1547-1548, 2008.

11. Tusher VG, Tibshirani R and Chu G: Significance analysis of microarrays applied to the ionizing radiation response. Proc Natl Acad Sci USA 98: 5116-5121, 2001.

12. Ohshima K, Kawasaki C, Muta H, et al: CD10 and Bcl10 expression in diffuse large B-cell lymphoma: CD10 is a marker of improved prognosis. Histopathology 39: 156-162, 2001.

13. Gambichler T, Skrygan M, Appelhans C, et al: Expression of human beta-defensins in patients with mycosis fungoides. Arch Dermatol Res 299: 221-224, 2007.

14. Browning JL, Dougas I, Ngam-ek A, et al: Characterization of surface lymphotoxin forms. Use of specific monoclonal antibodies and soluble receptors. J Immunol 154: 33-46, 1995.

15. Ware CF, Crowe PD, Grayson MH, Androlewicz MJ and Browning JL: Expression of surface lymphotoxin and tumor necrosis factor on activated T, B, and natural killer cells. J Immunol 149: 3881-3888, 1992.

16. Fu YX, Huang G, Wang Y and Chaplin DD: B lymphocytes induce the formation of follicular dendritic cell clusters in a lymphotoxin alpha-dependent fashion. J Exp Med 187: 1009-1018, 1998.

17. Ware CF: Network communications: lymphotoxins, LIGHT, and TNF. Annu Rev Immunol 23: 787-819, 2005.

18. Rennert PD, Browning JL, Mebius R, Mackay F and Hochman PS Surface lymphotoxin alpha/beta complex is required for the development of peripheral lymphoid organs. J Exp Med 184 1999-2006, 1996.

19. Tumanov AV, Kuprash DV and Nedospasov SA: The role of lymphotoxin in development and maintenance of secondary lymphoid tissues. Cytokine Growth Factor Rev 14: 275-288, 2003.

20. Dejardin E, Droin NM, Delhase M, et al: The lymphotoxin-beta receptor induces different patterns of gene expression via two NF-kappaB pathways. Immunity 17: 525-535, 2002.

21. Dejardin E: The alternative NF-kappaB pathway from biochemistry to biology: pitfalls and promises for future drug development. Biochem Pharmacol 72: 1161-1179, 2006.

22. Matsushima A, Kaisho T, Rennert PD, et al: Essential role of nuclear factor (NF)-kappaB-inducing kinase and inhibitor of kappaB (IkappaB) kinase alpha in NF-kappaB activation through lymphotoxin beta receptor, but not through tumor necrosis factor receptor I. J Exp Med 193: 631-636, 2001.

23. Hehlgans T, Stoelcker B, Stopfer P, et al: Lymphotoxin-beta receptor immune interaction promotes tumor growth by inducing angiogenesis. Cancer Res 62: 4034-4040, 2002.

24. Barkett M and Gilmore TD: Control of apoptosis by Rel/ NF-kappaB transcription factors. Oncogene 18: 6910-6924, 1999

25. Biswas DK, Martin KJ, McAlister C, et al: Apoptosis caused by chemotherapeutic inhibition of nuclear factor-kappaB activation. Cancer Res 63: 290-295, 2003.

26. Dhawan P, Su Y, Thu YM, et al: The lymphotoxin-beta receptor is an upstream activator of NF-kappaB-mediated transcription in melanoma cells. J Biol Chem 283: 15399-15408, 2008.

27. Haybaeck J, Zeller N, Wolf MJ, et al: A lymphotoxin-driven pathway to hepatocellular carcinoma. Cancer Cell 16: 295-308, 2009.
28. Gilmore TD: Multiple mutations contribute to the oncogenicity of the retroviral oncoprotein v-Rel. Oncogene 18: 6925-6937, 1999.

29. Häcker H and Karin M: Is NF-kappaB2/p100 a direct activator of programmed cell death? Cancer Cell 2: 431-433, 2002.

30. Martinez-Delgado B, Meléndez B, Cuadros M, et al: Expression profiling of T-cell lymphomas differentiates peripheral and lymphoblastic lymphomas and defines survival related genes. Clin Cancer Res 10: 4971-4982, 2004.

31. Martinez-Delgado B, Cuadros M, Honrado E, et al: Differential expression of NF-kappaB pathway genes among peripheral T-cell lymphomas. Leukemia 19: 2254-2263, 2005.

32. Ballester B, Ramuz O, Gisselbrecht C, et al: Gene expression profiling identifies molecular subgroups among nodal peripheral T-cell lymphomas. Oncogene 25: 1560-1570, 2006.

33. Bekiaris V, Gaspal F, Kim MY, et al: CD30 is required for CCL21 expression and CD4 T cell recruitment in the absence of lymphotoxin signals. J Immunol 182: 4771-4775, 2009.

34. Kallinich T, Muche JM, Qin S, Sterry W, Audring H and Kroczek RA: Chemokine receptor expression on neoplastic and reactive $\mathrm{T}$ cells in the skin at different stages of mycosis fungoides. J Invest Dermatol 121: 1045-1052, 2003.

35. Sokolowska-Wojdylo M, Wenzel J, Gaffal E, et al: Circulating clonal CLA(+) and CD4(+) T cells in Se'zary syndrome express the skin-homing chemokine receptors CCR4 and CCR10 as well as the lymph node-homing chemokine receptor CCR7. Br J Dermatol 152: 258-264, 2005.

36. Willemze R, Kerl H, Sterry W, et al: EORTC classification for primary cutaneous lymphomas: a proposal from the Cutaneous Lymphoma Study Group of the European Organization for Research and Treatment of Cancer. Blood 90: 354-371, 1997.

37. Müller A, Homey B, Soto H, et al: Involvement of chemokine receptors in breast cancer metastasis. Nature 410: 50-56, 2001.

38. von Andrian UH and Mempel TR: Homing and cellular traffic in lymph nodes. Nat Rev Immunol 3: 867-878, 2003.

39. Balkwill F: Cancer and the chemokine network. Nat Rev Cancer 4: 540-550, 2004.

40. Jones D, O'Hara C, Kraus MD, et al: Expression pattern of T-cellassociated chemokine receptors and their chemokines correlates with specific subtypes of T-cell non-Hodgkin lymphoma. Blood 96: 685-690, 2000

41. Ferenczi K, Fuhlbrigge RC, Pinkus J, Pinkus GS and Kupper TS: Increased CCR4 expression in cutaneous T cell lymphoma. J Invest Dermatol 119: 1405-1410, 2002

42. Narducci MG, Scala E, Bresin A, et al: Skin homing of Se'zary cells involves SDF-1-CXCR4 signaling and down-regulation of CD26/dipeptidylpeptidase IV. Blood 107: 1108-1115, 2006.

43. Tang HL and Cyster JG: Chemokine up-regulation and activated $\mathrm{T}$ cell attraction by maturing dendritic cells. Science 284 : 819-822, 1999

44. Wu M, Fang H and Hwang ST: Cutting edge: CCR4 mediates antigen-primed $\mathrm{T}$ cell binding to activated dendritic cells. J Immunol 167: 4791-4795, 2001 .

45. Edelson RL: Cutaneous T cell lymphoma: the helping hand of dendritic cells. Ann N Y Acad Sci 941: 1-11, 2001.

46. Homey B, Alenius H, Müller A, et al: CCL27-CCR10 interactions regulate $\mathrm{T}$ cell-mediated skin inflammation. Nat Med 8: $157-165,2002$.

47. Fujita Y, Abe R, Sasaki M, et al: Presence of circulating CCR $10^{+}$ $\mathrm{T}$ cells and elevated serum CTACK/CCL27 in the early stage of mycosis fungoides. Clin Cancer Res 12: 2670-2675, 2006.

48. Kagami S, Sugaya M, Minatani Y, et al: Elevated serum CTACK/CCL27 levels in CTCL. J Invest Dermatol 126: 1189-1191, 2006.

49. Berger CL, Tigelaar R, Cohen J, et al: Cutaneous T-cell lymphoma: malignant proliferation of T-regulatory cells. Blood 105: 1640-1647, 2005

50. Karube K, Aoki R, Sugita Y, et al: The relationship of FOXP3 expression and clinicopathological characteristics in adult T-cell leukemia/lymphoma. Mod Pathol 21: 617-625, 2008. 